\title{
Demonstration of Reduced Complexity Multi-band CAP Modulation using Xia-Pulses in Visible Light Communications
}

\author{
Paul Anthony Haigh and Izzat Darwazeh \\ Communications and Informations Systems Group, University College London, London, WC1E 6BT, UK \\ \{p.haigh;i.darwazeh\}@ucl.ac.uk
}

\begin{abstract}
This work proposes and demonstrates, for the first time, a new method for CAP modulation in VLC based on full-Nyquist Xia pulses. By receiver modification, we demonstrate a signification reduction in computational complexity by $>90 \%$.
\end{abstract}

OCIS codes: $060.2605,060.4080,060.4510$.

\section{Introduction}

Visible light communications (VLC) is an emerging technology with typical application in access networks, offering a complementary alternative to radio frequency technologies. VLC introduces $\sim 300 \mathrm{THz}$ of additional bandwidth to support high speed links. This is timely as, according to forecasts, mobile data traffic is expected to grow at a compound annual growth rate of $57 \%$ until at least 2021 [1]. State-of-the-art developments in VLC mostly focus on overcoming the limitations of the light-emitting diodes (LEDs) that are commonly used for illumination. The key limitation is their low modulation bandwidths which exhibit first-order low-pass responses restricted to several $\mathrm{MHz}$ [2]. Several modulation formats have established themselves as leading candidates towards achieving $\mathrm{Gb} / \mathrm{s}$ rates including orthogonal frequency division multiplexing (OFDM) [3] and carrier-less amplitude and phase modulation (CAP) [2]. In [2], CAP was shown to outperform OFDM in terms of achievable transmission speeds and bit error rates (BERs) when compared over the same physical link using the same test infrastructure. CAP utilises a Hilbert pair of pulse shaping filters to ensure that a complex signal can be represented in the same frequency space, separated by phase. Generally, the pulse shaping filters used are based on the square-root raised cosine (SRRC), which is a rootNyquist filter. CAP was later extended into a multi-band scheme ( $m$-CAP) that aimed to mitigate the low-pass response of the LEDs, reducing the attenuation experienced across each sub-band, across each of the $m$ sub-bands [4]. Such extension enabled significant gains in spectral efficiency at the cost of greatly increased computational complexity, which is a serious issue in $m$-CAP systems.

Two finite impulse response (FIR) filters are required for each sub-band at both the transmitter (due to the Hilbert pair, as mentioned) and also at the receiver, where matched filters are required due to the use of the root-Nyquist SRRC, giving a total requirement of $4 m$ FIR filters. Furthermore, clear dependence between filter length and BER performance has been demonstrated [5], where long filters offer substantially improved BER performance over truncated versions of the same filters. The computational and implementation complexity issue has been alleviated somewhat by confirmation that the transmitter pulses can be stored in a look-up table [6]. In this work, we develop this concept a step further by modification of the filter selection to a Nyquist pulse, the Xia pulse [7], meaning that a regular QAM receiver can be used and eliminating the need for a matched filter, substantially reducing the computational complexity of the link. We confirm by experiment that similar BER performance can be obtained for the proposed link, with the computational complexity, i.e. number of multiplications, reduced by more than $90 \%$.

\section{Test Setup}

The experimental test setup is illustrated in Fig. 1. Three independent data streams are modulated into the binary phase shift keying (BPSK) modulation format, and upsampled following [4] before being split into their real ( $\Re$ ) and imaginary (I) components. The data is then passed through a pulse shaping filter. In this work, we test two filters, firstly the traditional SRRC filter in matched configuration, and secondly the Xia pulse [7] in both matched (rootNyquist) and full-Nyquist pulse shapes, which is the pulse shape of interest in this work. The aim of omitting the matched filter from the receiver is to reduce computational complexity. When a full-Nyquist pulse is used, we show 


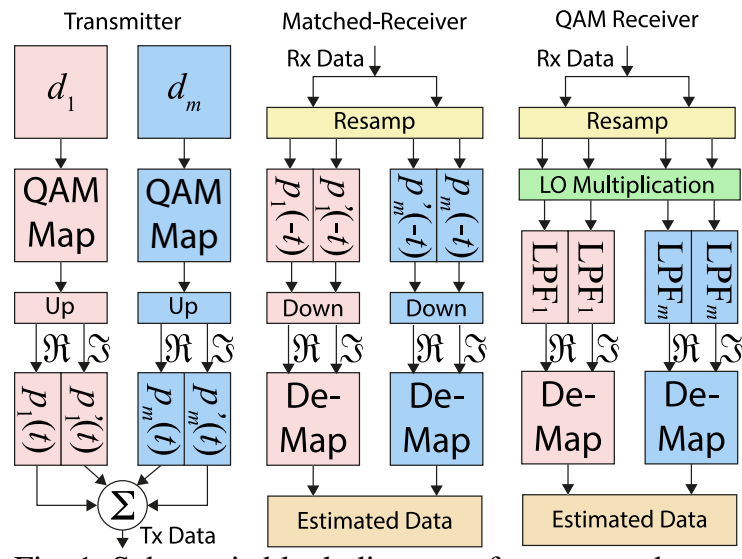

Fig. 1: Schematic block diagram of system under test

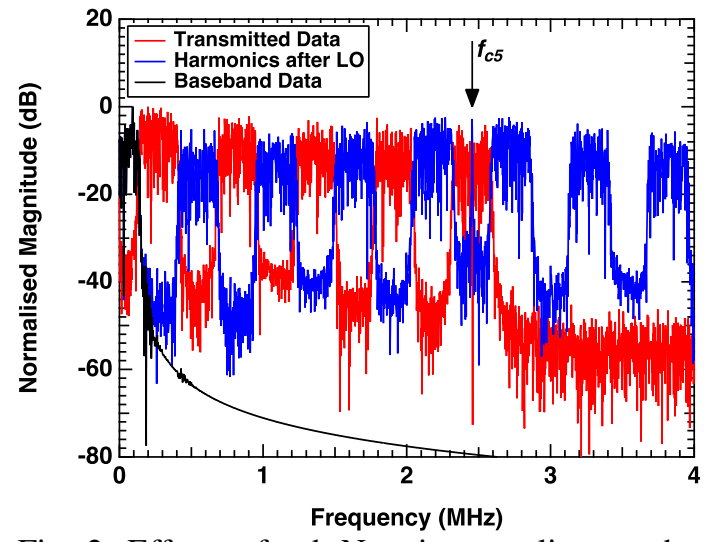

Fig. 2: Effects of sub-Nyquist sampling on the received signal after carrier removal of sub-band 5 .

that a typical QAM receiver can be utilised consisting only of a local-oscillator and low-pass filter. After pulse shaping, the $m$ sub-bands are summed before intensity modulation of the Osram Golden Dragon LED, driven at $550 \mathrm{~mA}$ in the linear region of its transfer function. The LED used is a high power white emitter suitable for indoor illumination with a measured bandwidth of $5 \mathrm{MHz}$ at $550 \mathrm{~mA}$ drive current and is modulated using a bias-tee. After transmission, a silicon PIN photodetector is used to generate an electrical signal before sampling via a Tektronix MDO4106-B real time oscilloscope. At the receiver, performed in MATLAB, the EVM is measured in the normal way [8] in order to estimate the SNR to find the appropriate number of bits/symbol for modulation of each sub-band for BER tests. The BER target is set to $3.8 \times 10^{-3}$ in line with the $7 \%$ forward error correction limit. The carrier frequency is set to odd integer multiples of the baud rate $R_{S}$ due to observation of sequentially reversed images at even harmonics after multiplication by the local oscillator due to sub-Nyquist band-pass sampling [9], causing a limitation in the placement of the sub-bands. This is illustrated in Fig. 2. The low-pass filter used is a $10^{\text {th }}$ order FIR filter, consisting of 11-taps.

The computational complexity (real valued multiplications/second) of the $m$-CAP link with matched filter configuration is given in [6] as $C_{m-C A P}=R_{S}\left(2 N+2 \sum_{n=1}^{N} M_{n} L\right)$, where $L$ is the filter length, $N$ is the total number of sub-bands and $M_{n}$ is the number of samples per symbol also given in $[4,6]$. Clearly the pulse shaping filters contribute the majority of the computational complexity, considering the $2 N$ component is introduced by the look-up tables of the transmit filters [6]. By using a low-pass filter in exchange for matched filters, it is possible to reduce the complexity of the proposed Xia-based $m$-CAP to $C_{m-C A P}=R_{s}\left(2 N+2 \sum_{n=1}^{N}\left(F_{L}+1\right)\right)$, where $F_{L}$ is the low-pass filter order, in this case set to 10 .

\section{Results}

The measured SNR for $m=10$ is shown in Fig. 3. It should be noted that SNR increases from $\sim 18 \mathrm{~dB}$ for 1-CAP to that shown in Fig. 3, as expected according to [8]. The SRRC matched filter configuration offers an SNR gain of $\sim 1.5 \mathrm{~dB}$. The root cause of this is down to the peak position of the Xia pulse shape in comparison to the SRRC. For Xia, unlike SRRC, the zero-crossing is not the peak which introduces a theoretical power penalty of approximately $0.5 \mathrm{~dB}$ in the best case, without considering sampling offset jitter [10], with constellations for the $4^{\text {th }}$ sub-bands shown.

The BER performance for each format is shown in Fig. 4 for $m=10$. At least $10^{6}$ bits were transmitted and tested. Clearly, each of the three links demonstrate a similar BER performance, where an optimal error free performance is offered for the $5^{\text {th }}$ sub-band, with constellations shown inset for SRRC (black), Xia with matched configuration (red) and the proposed Xia full Nyquist method (blue), each with 256-QAM. Each method meets the proposed BER target of $3.8 \times 10^{-6}$. The individual methods resulted in total BER performances (the ratio of the total sum of the errors and total bits) of $3.7 \times 10^{-4}, 1 \times 10^{-3}$ and $3.63 \times 10^{-4}$, for SRRC, Xia-matched and the proposed Xia-CAP, all meeting the BER target with adaptive bit-loading. Only the total BERs are described and are (i) $m=5,2.59 \times 10^{-4}$ (SRRC), $8.26 \times 10^{-4}$ (Xia matched) and $6.05 \times 10^{-4}$ (proposed Xia); (ii) $m=2,8 \times 10^{-5}$ (SRRC), $1.86 \times 10^{-4}$ (Xia matched) and $9.58 \times 10^{-4}$ (proposed Xia); and (iii) $m=1,1.25 \times 10^{-5}$ (SRRC), $6.5 e-5 \times 10^{-4}$ (Xia matched) and $9.73 \times 10^{-4}$ (proposed Xia). All of which meet the proposed BER target and demonstrate the effectiveness of the proposed receiver method, due to the similar BER performances.

In terms of computational complexity, for the system used, $L$ was set to 20 , and the number of samples/symbol was set dynamically according to the number of sub-bands following [4]. At a baud rate, of $R_{S}=2.7 \mathrm{MBd} / \mathrm{s}$, used 


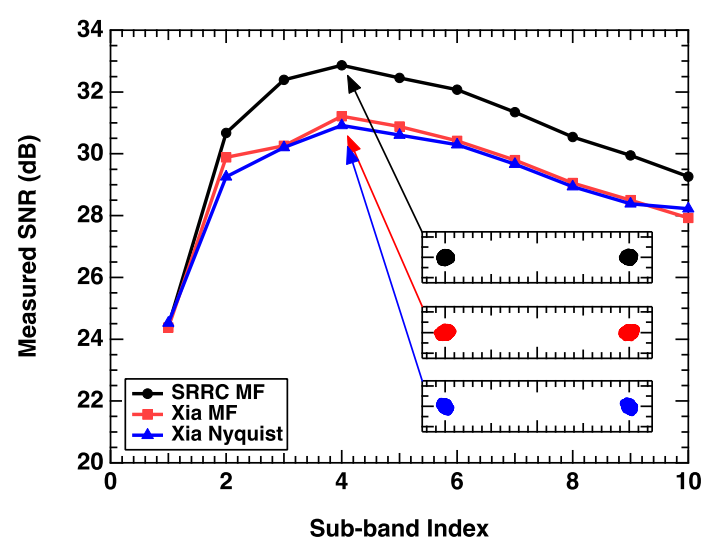

Fig. 3: Measured SNR of the 10-CAP systems

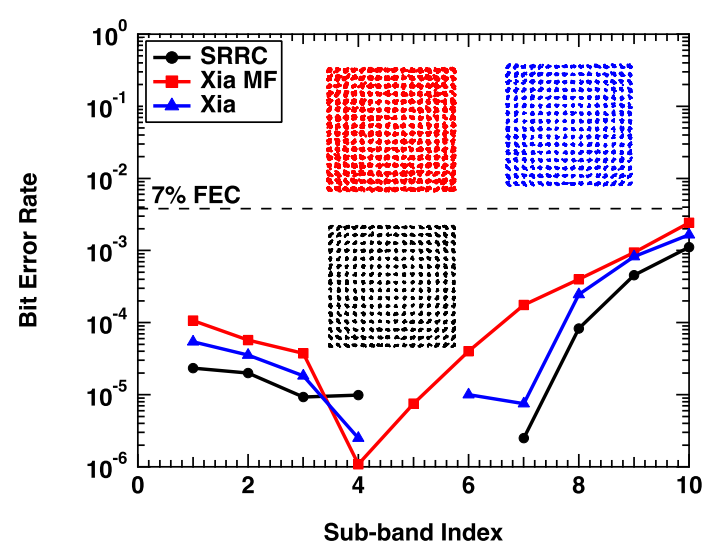

Fig. 4: Comparative 10-CAP BER performance

for the BPSK tests, with $3 \mathrm{MHz}$ total bandwidth, the minimum computational complexity of the receiver in matched configuration was for $N=1$, where $M_{n}=9$ and $C_{1-C A P}=R_{S}\left(2 N+2 M_{n} L\right)=9.556 \times 10^{8}$ mult $/ s$, which increases 10 -fold to $9.6 \times 10^{9} \mathrm{mult} / \mathrm{s}$ for $N=10$. Using the proposed receiver reduces the computational complexity by $>90 \%$ for $N=10$ to $6.48 \times 10^{8}$ mult $/ \mathrm{s}$, providing a substantial complexity gain with no relative loss in performance.

\section{Conclusion}

In this paper we have demonstrated a novel receiver structure for $m$-CAP for VLC systems. Previously, $m$-CAP has been shown to offer substantial performance gains over other modulation formats, but its main drawback has been its computational complexity. In this work, we replace the traditional CAP receiver with a modified structure based on full Nyquist pulses at the transmitter, and a local oscillator and low-pass filter at the receiver. This results in a decrease in computational complexity of $93.25 \%$.

\section{Acknowledgement}

This work is supported by UK EPSRC Grant EP/P006280/1: Multifunctional Polymer Light-Emitting Diodes with Visible Light Communications (MARVEL).

\section{References}

1. - Cisco Visual Network Index: Global Mobile Data Traffic Forecast, 2016-2021, 2017.

2. F.M. Wu, et al., Performance comparison of OFDM signal and CAP signal over high capacity RGB-LED-based WDM visible light communication., IEEE Photonics Journal, 2013.

3. Tsonev, D., et al., A 3-Gb/s single-LED OFDM-based wireless VLC link using a gallium nitride $\mu$ LED. IEEE Photon. Technol. Lett., 2014.

4. Olmedo, M.I., et al., Multiband carrierless amplitude phase modulation for high capacity optical data links, $J$. Lightw. Technol., 2014.

5. Chvojka, P., et al., Evaluation of Multi-band Carrier-less Amplitude and Phase Modulation Performance for VLC under Various Pulse Shaping Filter Parameters, 13th International Joint Conference on e-Business and Telecommunications, 2016.

6. Wei, J.L., et al., Fair comparison of complexity between a multi-band CAP and DMT for data center interconnects. Optics Letters, 2017.

7. Xia, X.G., A family of pulse-shaping filters with ISI-free matched and unmatched filter properties, IEEE Trans. Commun., 1997.

8. Haigh, P.A., et al., A multi-CAP visible-light communications system with $4.85-\mathrm{b} / \mathrm{s} / \mathrm{Hz}$ spectral efficiency, IEEE J. Sel. Areas in Commun., 2015.

9. M. Patel, I. Darwazeh and J. J. O'Reilly, "Bandpass sampling for software radio receivers, and the effect of oversampling on aperture jitter,' Vehicular Technology Conference. IEEE Vehicular Technology Conference, 2002.

10. C. C. Tan and N. C. Beaulieu, "Transmission properties of conjugate-root pulses," IEEE Trans. Commun., 2004. 